\title{
An inverse spectral problem on surfaces
}

\author{
Philippe Castillon
}

\begin{abstract}
The purpose of this paper is to prove how the positivity of some operators on a Riemannian surface gives informations on the conformal type of the surface (the operators considered here are of the form $\Delta+\lambda \mathcal{K}$ where $\Delta$ is the Laplacian of the surface, $\mathcal{K}$ is its curvature and $\lambda$ is a real number). In particular we obtain a theorem "à la Huber": under a spectral hypothesis we prove that the surface is conformally equivalent to a Riemann surface with a finite number of points removed.

This problem has its origin in the study of stable minimal surfaces.

Résumé. On montre dans cet article comment la positivité de certains opérateurs sur une surface riemannienne permet d'obtenir des informations sur le type conforme de la surface (les opérateurs considérés ici sont de la forme $\Delta+\lambda \mathcal{K}$ où $\Delta$ est le laplacien sur la surface, $\mathcal{K}$ sa courbure et $\lambda$ un réel). On montre en particulier un théorème "à la Huber" : partant d'une hypothèse spectrale, on en déduit que la surface est conformément équivalente à une surface de Riemann compacte privée d'un nombre fini de points.

Ce problème trouve son origine dans l'étude des surfaces minimales stables.
\end{abstract}

Mathematics Subject Classification (2000). 58J50, 53A30, 53A10.

Keywords. Spectral theory, minimal surfaces, stability operator.

\section{Introduction}

Minimal submanifolds are solutions of a variational problem: they are critical points of the volume functional for deformations with compact support. The second derivative of the volume functional is given by a quadratic form associated to a selfadjoint operator (the stability operator). A minimal immersion is called stable when it is a local minimum of the volume functional, that is, when the stability operator is nonnegative.

For a minimal surface $M$ in $\mathbb{R}^{3}$, the stability operator is given by $S=\Delta+2 \mathcal{K}$, where $\mathcal{K}$ is the (intrinsic) curvature of $M$. For a surface immersed in a manifold with nonnegative scalar curvature, the positivity of the stability operator implies the positivity of the operator $L=\Delta+\mathcal{K}$. In order to study stable minimal surfaces, these remarks lead the authors of [FC-Sc] to consider the problem of relating the 
positivity of operators of the form $\Delta+\lambda \mathcal{K}(\lambda \in \mathbb{R})$ on a surface to the geometry of the surface.

Let $(M, h)$ be a complete noncompact Riemannian surface and let $\mathcal{K}$ be its curvature. For all $\lambda \in \mathbb{R}$, consider the operator $L_{\lambda}=\Delta+\lambda \mathcal{K}$ and the associated quadratic form $q_{\lambda}$. It is easy to see (cf. [FC-Sc]) that the set $I_{h}=\left\{\lambda \in \mathbb{R} \mid q_{\lambda}\right.$ positive $\}$ is a closed interval: $I_{h}=\left[a_{h}, b_{h}\right]$ with $-\infty \leq a_{h} \leq 0 \leq b_{h} \leq+\infty$. The general problem is to find relations between the geometry of $M$ and the numbers $a_{h}$ and $b_{h}$.

In [FC-Sc] the authors asked the following question: On the disc $D=\{z \in \mathbb{C} \mid$ $|z|<1\}$, consider the complete metrics which are conformal to the Euclidean one; for such a metric $h$, what are the possible values of $b_{h}$ which can occur? As a first step to answer this question, they remark that $b_{h}=\frac{1}{4}$ if $h$ is the Poincaré metric and they prove that $b_{h}<1$ for a complete conformal metric on $D$ (cf. [FC-Sc], Remark 1 and Theorem 2). One of the purpose of this paper is to answer this question (cf. Section 4).

In the more general context of a complete noncompact Riemannian surface we prove the following result.

Theorem A. Let $(M, h)$ be a complete noncompact Riemannian surface. If $b_{h}>\frac{1}{4}$ then $M$ is conformally equivalent to $\mathbb{C}$ or $\mathbb{C}^{*}=\mathbb{C} \backslash\{0\}$.

A straightforward corollary is that $b_{h} \leq \frac{1}{4}$ when $h$ is a complete conformal metric on the disc. Moreover, with a similar proof we get the following theorem which is to be compared with Huber's theorem (cf. [Hu]).

Theorem B. Let $(M, h)$ be a complete noncompact Riemannian surface. If there exists a compact domain $\Omega \in M$ and a real $\lambda>\frac{1}{4}$ such that $q_{\lambda}(u) \geq 0$ for every $C^{\infty}$-function $u$ with compact support in $M \backslash \Omega$, then $M$ is conformally equivalent to a compact Riemann surface with a finite number of points removed.

In the first section we precise the notations and give preliminary results. The second and third sections are devoted to the proofs of Theorem A and Theorem B respectively. In the fourth section we treat the particular case of complete conformal metrics on the unit disc.

The main results of this paper were already announced without proof in [Ca2].

\section{Notations and preliminary results}

Let $(M, h)$ be a surface endowed with a Riemannian metric $h$; we note $\mathcal{K}$ its curvature and $d v_{h}$ its volume form. For a real number $\lambda$, we note $L_{\lambda}$ the operator $L_{\lambda}=\Delta+\lambda \mathcal{K}$ acting on the space $C_{0}^{\infty}(M)$ of compactly supported $C^{\infty}$ functions (where $\Delta$ is the positive Laplacian); each of these operators admits a unique self-adjoint extension 
(also denoted $L_{\lambda}$ ). We shall note $q_{\lambda}$ the associated quadratic form; for all $u \in C_{0}^{\infty}(M)$, we have $q_{\lambda}(u)=\int_{M}\left(|d u|_{h}^{2}+\lambda \mathcal{K} u^{2}\right) d v_{h}$.

In order to study the relations between the positivity of some of the forms $q_{\lambda}$ and the geometry of $M$, we introduce the set $I_{h}=\left\{\lambda \in \mathbb{R} \mid q_{\lambda}\right.$ is nonnegative $\}$.

Proposition 1.1. The set $I_{h}$ is a closed interval of $\mathbb{R}$ containing 0: $I_{h}=\left[a_{h}, b_{h}\right]$ with $-\infty \leq a_{h} \leq 0 \leq b_{h} \leq+\infty$.

Proof. Let $F(\lambda)=\inf \left\{q_{\lambda}(u) \mid u \in C_{0}^{\infty}(M)\right\}$; the set $I_{h}$ is just the subset of $\mathbb{R}$ where $F$ is nonnegative. Since $F$ is the infimum of affine functions, it must be concave, and the proposition follows.

Example 1.2. It is easy to see that $b_{h}=+\infty$ if and only if $\mathcal{K} \geq 0$ on $M$, and that $a_{h}=-\infty$ if and only if $\mathcal{K} \leq 0$ on $M$.

Example 1.3. Let $D=\{z \in \mathbb{C}|| z \mid<1\}$, and let $h_{\mathbb{H}^{2}}$ be the Poincaré metric on $D$. It is a well-known fact that the spectrum of the Laplacian is $\sigma(\Delta)=\left[\frac{1}{4}, \infty\right)$. Since the curvature is constant and equal to -1 , we have $\sigma(\Delta+\lambda \mathcal{K})=\left[\frac{1}{4}-\lambda, \infty\right)$. It follows that $b_{h_{\mathbb{H}^{2}}}=\frac{1}{4}$ in this case.

Doing the same for an arbitrary hyperbolic surface $(M, h)$, we have $b_{h}=\inf (\sigma(\Delta))$.

Notations. Let $x_{0}$ be a point in $M$. In the sequel we shall note $r(x)=d_{M}\left(x_{0}, x\right)$ the distance function to $x_{0}, B_{s}=\{x \in M \mid r(x)<s\}$ the ball of radius $s$, and $C_{s}^{t}=\{x \in M \mid s<r(x)<t\}$.

Moreover, we shall note $V(s)$ the volume of the ball $B_{s}, \ell(s)$ the length of the geodesic circle of radius $s$ (i.e. $\left.\ell(s)=\operatorname{vol}\left(\partial B_{s}\right)\right)$ and $G(s)$ the total curvature of the ball $B_{s}$ (i.e. $G(s)=\int_{B_{s}} \mathcal{K} d v_{h}$ ). Using the coarea formula, we easily have $V^{\prime}(s)=\ell(s)$ and $G^{\prime}(s)=\int_{\partial B_{s}} \mathcal{K} d \sigma_{s}$ where $d \sigma_{s}$ is the volume form on $\partial B_{s}$.

Topology of noncompact surfaces. For a compact surface $S$ we shall note $g_{S}$ its genus and $n_{S}$ the number of connected components of its boundary; in particular, the Euler characteristic of $S$ is given by $\chi(S)=2\left(1-g_{S}\right)-n_{S}$.

A noncompact surface $S$ is said to be of finite topology if there exists a compact surface $\widetilde{S}$ without boundary and a finite number of pairwise disjoint closed discs $D_{i}$, $i=1, \ldots, N$, in $\widetilde{S}$ so that $S$ is homeomorphic to $\widetilde{S} \backslash \bigcup_{i=1}^{N} D_{i}$ (i.e. $S$ is homeomorphic to the interior of a compact surface with boundary). In this case, we define the Euler characteristic of $S$ to be $\chi(S)=\chi\left(\widetilde{S} \backslash \bigcup_{i=1}^{N} \stackrel{\circ}{D}{ }_{i}\right)=2(1-g \widetilde{S})-N$.

For a complete Riemannian surface $(M, h)$, we have the following relations between the asymptotic behaviour of $\chi\left(B_{S}\right)$ and the topology of $M$ : 
Lemma 1.4. Let $(M, h)$ be a complete Riemannian surface.

(i) If $M$ is of finite topology, then there exists $s_{0}$ such that for all $s \geq s_{0}$ we have $\chi\left(B_{s}\right) \leq \chi(M)$

(ii) If $M$ is not of finite topology then $\lim _{s \rightarrow \infty} \chi\left(B_{s}\right)=-\infty$.

Proof. Suppose that $M$ is of finite topology. Then there exists a compact surface $\tilde{M}$, a finite set $\left\{p_{1}, \ldots, p_{N}\right\} \subset \widetilde{M}$ and a homeomorphism $f: M \rightarrow \widetilde{M} \backslash\left\{p_{1}, \ldots, p_{N}\right\}$. Let $\widetilde{U}_{1}, \ldots, \widetilde{U}_{N}$ be simply connected open neighborhoods of the points $p_{1}, \ldots, p_{N}$ which are pairwise disjoint, let $\widetilde{C}=\widetilde{M} \backslash \bigcup_{i=1}^{N} \widetilde{U}_{i}$, and let $C=f^{-1}(\widetilde{C})$. The set $C$ is a compact domain in $M$, and by construction we have $\chi(C)=\chi(\widetilde{C})=\chi(M)$.

Choose $s_{0}$ such that $C \subset \stackrel{\circ}{B}_{s_{0}}$; for all $s \geq s_{0}$, we have $C \subset B_{s}$, which implies that

$$
g_{C} \leq g_{B_{s}} .
$$

For all $s \geq s_{0}$, note $\widetilde{B}_{s}=f\left(B_{s}\right)$. Since $C \subset \stackrel{\circ}{B}_{s}$ we have $\partial \widetilde{B}_{s} \subset \widetilde{M} \backslash \widetilde{C}$, and since $\widetilde{B}_{s}$ is compact in $\widetilde{M} \backslash\left\{p_{1}, \ldots, p_{N}\right\}$, we also have $\partial \widetilde{B}_{S} \cap \widetilde{U}_{i} \neq \emptyset$ for all $i \in\{1, \ldots, N\}$. It follows that

$$
n_{B_{s}}=n_{\widetilde{B}_{s}} \geq N=n_{C} .
$$

From the inequalities (1.1) and (1.2) we deduce that for all $s \geq s_{0}$ we have

$$
\chi\left(B_{s}\right) \leq \chi(C)=\chi(M) .
$$

To prove (ii), we suppose that $\chi\left(B_{s}\right)$ does not tend to $-\infty$, and we shall prove that the topology of $M$ is finite. Using the hypothesis, there exists a constant $A>0$ and an increasing sequence $\left(s_{i}\right)_{i \in \mathbb{N}}$ such that $\lim _{i \rightarrow \infty} s_{i}=+\infty$ and $\chi\left(B_{S_{i}}\right) \geq-A$ for all $i \in \mathbb{N}$. In particular we have $2\left(1-g_{B_{s_{i}}}\right)-n_{B_{s_{i}}} \geq-A$, from which we deduce that $g_{B_{s_{i}}} \leq \frac{A+2}{2}$ and $n_{B_{s_{i}}} \leq A+2$.

For all $i \in \mathbb{N}$, note $E_{i, j}, j=1, \ldots, a_{i}$ the compact connected components of $M \backslash B_{S_{i}}$. We construct an exhaustion of $M$ by compact domains in the following way:

- $\Omega_{0}=B_{s_{0}} \cup\left(\bigcup_{j=1}^{a_{0}} E_{0, j}\right)$.

- Suppose that $\Omega_{0}, \ldots, \Omega_{k}$ are known; let $i_{k}$ such that $\Omega_{k} \subset \stackrel{\circ}{B}_{s_{i_{k}}}$, and let $\Omega_{k+1}=$ $B_{s_{i_{k}}} \cup\left(\bigcup_{j=1}^{a_{i_{k}}} E_{i_{k}, j}\right)$.

By construction, $\Omega_{k}$ is connected and $M \backslash \Omega_{k}$ has no compact connected component. Moreover, for all $k \in \mathbb{N}$ we have $g_{\Omega_{k}} \leq g_{B_{s_{i}}} \leq \frac{A+2}{2}$ and $n_{\Omega_{k}} \leq n_{B_{s_{i k-1}}} \leq A+2$. From these inequalities we deduce that there exists a constant $A_{1}>0$ such that for all $k \in \mathbb{N}$ we have

$$
\chi\left(\Omega_{k}\right)=2\left(1-g_{\Omega_{k}}\right)-n_{\Omega_{k}} \geq-A_{1} .
$$


For all $k \in \mathbb{N}$ write $\Omega_{k+1} \backslash \Omega_{k}=\bigcup_{j=1}^{b_{k}} F_{k, j}$ where the domains $F_{k, j}$ are the connected components of $\Omega_{k+1} \backslash \Omega_{k}$. For all $k$ and all $j=1, \ldots, b_{k}, \partial F_{k, j}$ has at least two connected component: one in $\partial \Omega_{k}$ because $\Omega_{k+1}$ is connected and one in $\partial \Omega_{k+1}$ since $M \backslash \Omega_{k}$ has no compact connected component. It follows that $\chi\left(F_{k, j}\right) \leq 0$, and since

$$
\chi\left(\Omega_{k+1}\right)=\chi\left(\Omega_{k}\right)+\sum_{j=1}^{b_{k}} \chi\left(F_{k, j}\right),
$$

we have $\chi\left(\Omega_{k+1}\right) \leq \chi\left(\Omega_{k}\right)$. From the inequality (1.3) we deduce that there exists $k_{0} \in \mathbb{N}$ such that $\chi\left(\Omega_{k}\right)=\chi\left(\Omega_{k_{0}}\right)$ for all $k \geq k_{0}$. On the other hand, since the sequence $\left(g_{\Omega_{k}}\right)_{k}$ is increasing and bounded, we can assume (up to a change of $k_{0}$ ), that $g_{\Omega_{k}}=g_{\Omega_{k_{0}}}$ for all $k \geq k_{0}$; it follows that $\Omega_{k}$ and $\Omega_{k_{0}}$ are homeomorphic for all $k \geq k_{0}$.

Let $k \geq k_{0}$; from equality (1.4) we deduce that $\sum_{j=1}^{b_{k}} \chi\left(F_{k, j}\right)=0$, and since $\chi\left(F_{k, j}\right) \leq 0$ for all $j=1, \ldots, b_{k}$ we have $\chi\left(F_{k, j}\right)=0$, which implies that $\bigcup_{j=1}^{b_{k}} F_{k, j}$ is homeomorphic to $\left.\left.\partial \Omega_{k_{0}} \times\right] 0,1\right]$.

Let $\tilde{M}$ be the surface obtained by gluing $\Omega_{k_{0}}$ and $\partial \Omega_{k_{0}} \times[0,1[$ along their boundaries $\partial \Omega_{k_{0}}$ and $\partial \Omega_{k_{0}} \times\{0\}$. Let $\left(c_{k}\right)_{k \geq k_{0}}$ be an increasing sequence of real numbers which tends to 1 and such that $c_{k_{0}}=0$, and let $\widetilde{\Omega}_{k}=\Omega_{k_{0}} \cup \partial \Omega_{k_{0}} \times\left[0, c_{k}\right] \subset \widetilde{M}$. We construct a sequence of homeomorphisms $f_{k}: \Omega_{k} \rightarrow \widetilde{\Omega}_{k}$ in the following way:

- $f_{k_{0}}: \Omega_{k_{0}} \rightarrow \widetilde{\Omega}_{k_{0}}$ is the identity;

- if $f_{k}$ is known, we extend it to a homeomorphism $f_{k+1}: \Omega_{k+1} \rightarrow \widetilde{\Omega}_{k+1}$ (which is possible since $\Omega_{k+1} \backslash \Omega_{k}$ is homeomorphic to $\left.\left.\partial \Omega_{k_{0}} \times\right] c_{k}, c_{k+1}\right]$ ).

This construction gives a homeomorphism $f: M \rightarrow \widetilde{M}$ defined by $f_{\mid \Omega_{k}}=f_{k}$ for all $k \geq k_{0}$, and the surface $\widetilde{M}$ is of finite topology, which proves item (ii) of the lemma.

Remark 1.5. Lemma 1.4 holds if we replace the geodesic balls of the metric by an exhaustion of $M$ by a sequence of compact domains (and the proof is the same).

In the proof of item (ii), the construction of the homeomorphism from its restrictions to compact domains is a classical tool in the theory of surfaces (cf. for example the proof of Kerékjártó's theorem on the topological classification of noncompact surfaces, Theorem 1 in [Ri]).

Remark 1.6. When $M$ is of finite topology, we may have $\chi\left(B_{s}\right)<\chi(M)$ for arbitrarily large $s$. Moreover, the converse statement of item (ii) is false.

The length of geodesic circles. The regularity properties of the function $\ell$ were already studied, in particular in connection to the isoperimetric problem (cf. [Fi], [Ha], [Sh-Ta1], [Sh-Ta2]). In general, the function $\ell$ is not continuous, however we have the following result: 
Theorem 1.7. The function $\ell$ is differentiable almost everywhere and we have

(i) for almost all $s \in \mathbb{R}, \ell^{\prime}(s) \leq 2 \pi \chi\left(B_{s}\right)-G(s)$;

(ii) for all $0 \leq a<b, \ell(b)-\ell(a) \leq \int_{a}^{b} \ell^{\prime}(s) d s$.

Proof. Cf. [Sh-Ta1] and [Sh-Ta2].

A technical lemma. In the proofs of Theorem A and B, we will evaluate the quadratic forms $q_{\lambda}$ on functions of the form $\xi(r)$, where $r$ is the distance function on $M$. To do this, we have to handle with terms of the form $\int_{M} \mathcal{K} \xi(r)^{2} d v_{h}$ which will be estimated using the following lemma. The proof of this lemma is based on the method used by T. Colding and W. Minicozzi in [Co-Mi].

Lemma 1.8. Let $R<Q$, and let $\xi:[R, Q] \rightarrow \mathbb{R}$ such that $\xi(Q)=0, \xi \geq 0, \xi^{\prime} \leq 0$ and $\xi^{\prime \prime} \geq 0$. If there exists a constant $A$ such that $\chi\left(B_{s}\right) \leq$ A for all $s \in[R, Q]$, then $\int_{C_{R}^{Q}} \mathcal{K} \xi(r)^{2} d v_{h} \leq-\xi(R)^{2} G(R)-2 \xi(R) \xi^{\prime}(R) \ell(R)+2 \pi A \xi(R)^{2}-\int_{C_{R}^{Q}}\left(\xi^{2}\right)^{\prime \prime}(r) d v_{h}$.

Proof. Using the coarea formula we have

$$
\int_{C_{R}^{Q}} \mathcal{K} \xi(r)^{2} d v_{h}=\int_{R}^{Q} \xi(s)^{2}\left(\int_{\partial B_{s}} \mathcal{K} d \sigma_{s}\right) d s=\int_{R}^{Q} \xi(s)^{2} G^{\prime}(s) d s .
$$

Let $H(s)=\int_{R}^{s} G(t) d t$. Doing two integrations by parts we get

$$
\begin{aligned}
\int_{C_{R}^{Q}} \mathcal{K} \xi(r)^{2} d v_{h} & =\left[\xi(s)^{2} G(s)\right]_{R}^{Q}-\int_{R}^{Q}\left(\xi^{2}\right)^{\prime}(s) G(s) d s \\
& =-\xi(R)^{2} G(R)-\left[\left(\xi^{2}\right)^{\prime}(s) H(s)\right]_{R}^{Q}+\int_{R}^{Q}\left(\xi^{2}\right)^{\prime \prime}(s) H(s) d s \\
& =-\xi(R)^{2} G(R)+\int_{R}^{Q}\left(\xi^{2}\right)^{\prime \prime}(s) H(s) d s .
\end{aligned}
$$

By Theorem 1.7 we have $G(t) \leq 2 \pi A-\ell^{\prime}(t)$, and therefore

$$
H(s) \leq 2 \pi A(s-R)-\int_{R}^{s} \ell^{\prime}(t) d t \leq 2 \pi A(s-R)+\ell(R)-\ell(s) .
$$

Since $\left(\xi^{2}\right)^{\prime \prime} \geq 0$, we get

$$
\begin{aligned}
\int_{C_{R}^{Q}} \mathcal{K} \xi(r)^{2} d v_{h} \leq- & \xi(R)^{2} G(R)+2 \pi A \int_{R}^{Q}\left(\xi^{2}\right)^{\prime \prime}(s)(s-R) d s \\
& +\ell(R) \int_{R}^{Q}\left(\xi^{2}\right)^{\prime \prime}(s) d s-\int_{R}^{Q}\left(\xi^{2}\right)^{\prime \prime}(s) \ell(s) d s .
\end{aligned}
$$


Doing an integration by part and using the coarea formula we get the desired inequality:

$$
\begin{aligned}
& \int_{C_{R}^{Q}} \mathcal{K} \xi(r)^{2} d v_{h} \\
& \leq-\xi(R)^{2} G(R)+2 \pi A\left[\left(\xi^{2}\right)^{\prime}(s)(s-R)\right]_{R}^{Q} \\
& \quad-2 \pi A \int_{R}^{Q}\left(\xi^{2}\right)^{\prime}(s) d s+\ell(R)\left[\left(\xi^{2}\right)^{\prime}(s)\right]_{R}^{Q}-\int_{R}^{Q}\left(\xi^{2}\right)^{\prime \prime}(s) \ell(s) d s \\
& \quad \leq-\xi(R)^{2} G(R)+2 \pi A \xi(R)^{2}-2 \xi(R) \xi^{\prime}(R) \ell(R)-\int_{C_{R}^{Q}}\left(\xi^{2}\right)^{\prime \prime}(r) d v_{h} .
\end{aligned}
$$

A result of potential theory. In order to determine the conformal type of the ends of $M$, we will use the following result whose proof is based on the proof of Theorem 1.2 in $[\mathrm{Co}-\mathrm{Mi}]$.

Proposition 1.9. Let $X^{d}$ be a complete Riemannian manifold of dimension $d$ such that the volume of the geodesic balls of radius $R$ satisfies $V_{X}(R) \leq c R^{2}$ and let $\phi \in L_{\mathrm{loc}}^{1}(X)$ be a function which is nonnegative almost everywhere. If the operator $\Delta_{X}-\phi$ is nonnegative then the function $\phi$ is equal to 0 almost everywhere.

Proof. The idea of the proof is to use the volume estimate to compare the manifold $X$ with $\mathbb{R}^{2}$.

Let $x_{0} \in X$ be a fixed point; in this proof, we shall note $r_{X}(x)=d_{X}\left(x_{0}, x\right), B_{s}$ the ball of radius $s$ centered in $x_{0}, V_{X}(s)$ the volume of $B_{s}, \ell_{X}(s)$ the volume of $\partial B_{s}$, and $C_{s}^{t}=\left\{x \in X \mid s<r_{X}(x)<t\right\}$.

In the same way, for $x \in \mathbb{R}^{2}$ we shall note $\tilde{r}(x)=|x|, \widetilde{B}_{s}$ the Euclidean ball of radius $s, \widetilde{V}(s)$ the volume of $\widetilde{B}_{s}, \tilde{\ell}(s)$ the volume of $\partial \widetilde{B}_{s}$ and $\widetilde{C}_{s}^{t}=\left\{x \in \mathbb{R}^{2} \mid s<\right.$ $\tilde{r}(x)<t\}$. The hypothesis implies that $V_{X}(s) \leq a \widetilde{V}(s)$ for some constant $a$.

For $Q>0$, let $\xi_{Q}(s)=\int_{s}^{Q} \frac{d t}{t}$; up to a multiplicative constant, $\xi(\tilde{r})$ is the Green function of the ball of radius $Q$ in $\mathbb{R}^{2}$ and we have $\widetilde{\Delta} \xi(\tilde{r})=0$ on $\mathbb{R}^{2} \backslash\{0\}$.

Let $R>0$, and let $\eta:[0, Q] \rightarrow \mathbb{R}$ the function defined by

$$
\eta(s)= \begin{cases}\xi_{Q}(R) & \text { on }[0, R[ \\ \xi_{Q}(s) & \text { for all } s \in[R, Q] .\end{cases}
$$

Let $p$ be the quadratic form associated to the operator $\Delta-\phi$. Since $p(\eta(r)) \geq 0$ and since $\phi$ is nonnegative almost everywhere, we have

$$
\xi_{Q}(R)^{2} \int_{B_{R}} \phi d v_{X} \leq \int_{C_{R}^{Q}}\left|d \xi_{Q}(r)\right|^{2} d v_{X}=\int_{R}^{Q} \xi_{Q}^{\prime}(s)^{2} \ell_{X}(s) d s .
$$


Since $\ell_{X}=V_{X}^{\prime}$, an integration by parts gives

$$
\xi_{Q}(R)^{2} \int_{B_{R}} \phi d v_{X} \leq\left[\xi_{Q}^{\prime}(s)^{2} V_{X}(s)\right]_{R}^{Q}-\int_{R}^{Q} 2 \xi_{Q}^{\prime}(s) \xi_{Q}^{\prime \prime}(s) V_{X}(s) d s .
$$

Using the upper bound on $V_{X}$ and doing another integration by part we have

$$
\begin{aligned}
\xi_{Q}(R)^{2} \int_{B_{R}} \phi d v_{X} & \leq\left[\xi_{Q}^{\prime}(s)^{2} V_{X}(s)\right]_{R}^{Q}-a \int_{R}^{Q} 2 \xi_{Q}^{\prime}(s) \xi_{Q}^{\prime \prime}(s) \widetilde{V}(s) d s \\
& \leq\left[\xi_{Q}^{\prime}(s)^{2}\left(V_{X}(s)-a \tilde{V}(s)\right)\right]_{R}^{Q}+\int_{R}^{Q} \xi_{Q}^{\prime}(s)^{2} \tilde{\ell}(s) d s \\
& \leq a \pi+\int_{\tilde{C}_{R}^{Q}}\left|d \xi_{Q}(\tilde{r})\right|^{2} d \tilde{v},
\end{aligned}
$$

where we used that $V_{X}(Q) \leq a \widetilde{V}(Q)$ and the fact that $\widetilde{V}(R)=\pi R^{2}$. From Green's formula in $\mathbb{R}^{2}$ we deduce

$$
\begin{aligned}
\xi_{Q}(R)^{2} \int_{B_{R}} \phi d v_{X} & \leq a \pi+a \int_{\partial \widetilde{C}_{R}^{Q}} \xi_{Q}(\tilde{r})\left\langle\widetilde{\nabla} \xi_{Q}(\tilde{r}), v\right\rangle d \tilde{\sigma}+\int_{\widetilde{C}_{R}^{Q}} \xi_{Q}(\tilde{r}) \widetilde{\Delta} \xi_{Q}(\tilde{r}) d \tilde{v} \\
& \leq a \pi-a \xi_{Q}(R) \xi_{Q}^{\prime}(R) \tilde{\ell}(R)
\end{aligned}
$$

where $v$ is the exterior normal vector to $\widetilde{C}_{R}^{Q}$ and $d \tilde{\sigma}$ is the volume form on $\partial \widetilde{C}_{R}^{Q}$. Finally we get

$$
\int_{B_{R}} \phi d v_{X} \leq \frac{a \pi}{\xi_{Q}(R)^{2}}+\frac{a \tilde{\ell}(R)}{R \xi_{Q}(R)}
$$

and letting $Q$ tend to $+\infty$ ( $X$ is complete) we obtain $\int_{X} \phi d v_{X} \leq 0$, which gives the desired result since $\phi$ is nonnegative almost everywhere.

Remark 1.10. There is a similar result on surfaces which are conformally equivalent to a compact Riemann surface with a finite number of points removed: if $\phi$ is a nonnegative function and if the operator $\Delta-\phi$ is nonnegative, then $\phi$ must be zero almost everywhere. This is a consequence of the conformal invariance of the Dirichlet integral in dimension 2 (cf. [Bé-Be], Appendix 1).

\section{Proof of Theorem A}

Let $(M, h)$ be a Riemannian surface such that $b_{h}>\frac{1}{4}$. In particular, for $\lambda=b_{h}$, the quadratic form $q_{\lambda}$ is nonnegative. The proof of Theorem A splits into three parts. 


\section{The topology of the surface}

Proposition 2.1. Under the hypothesis of Theorem $A, M$ is homeomorphic to $\mathbb{C}$ or $\mathbb{C}^{*}=\mathbb{C} \backslash\{0\}$.

Proof. Suppose that $M$ is neither homeomorphic to $\mathbb{C}$, nor to $\mathbb{C}^{*}$; then we must be in one of the two following cases:

- $M$ is of finite topology and $\chi(M) \leq-1$;

- $M$ is of infinite topology.

In these two cases, by Lemma 1.4, there exists $R$ such that for all $s \geq R$ we have $\chi\left(B_{S}\right) \leq-1$.

Let $Q>R$ and let $\xi:[0, Q] \rightarrow \mathbb{R}$ be the function defined by

$$
\xi(s)= \begin{cases}(Q-R)^{\alpha} & \text { on }[0, R[ \\ (Q-s)^{\alpha} & \text { for all } s \in[R, Q],\end{cases}
$$

where $\alpha \geq 1$ will be chosen later.

Since the form $q_{\lambda}$ is nonnegative, we have

$$
\begin{aligned}
0 & \leq \int_{B_{Q}}\left(\xi^{\prime}(r)^{2}+\lambda \mathcal{K} \xi(r)^{2}\right) d v_{h} \\
& \leq \lambda \xi(R)^{2} G(R)+\int_{C_{R}^{Q}} \xi^{\prime}(r)^{2} d v_{h}+\lambda \int_{C_{R}^{Q}} \mathcal{K} \xi(r)^{2} d v_{h} .
\end{aligned}
$$

Since $\chi\left(B_{s}\right) \leq-1$ for all $s \in[R, Q]$, Lemma 1.8 gives

$$
\begin{aligned}
0 \leq \lambda \xi(R)^{2} G(R)+\int_{C_{R}^{Q}} \xi^{\prime}(r)^{2} d v_{h}-\lambda \xi(R)^{2} G(R)-2 \lambda \xi(R) \xi^{\prime}(R) \ell(R) \\
\quad-2 \pi \lambda \xi(R)^{2}-\lambda \int_{C_{R}^{Q}}\left(\xi^{2}\right)^{\prime \prime}(r) d v_{h} \\
\leq-2 \lambda \xi(R) \xi^{\prime}(R) \ell(R)-2 \pi \lambda \xi(R)^{2} \\
+\int_{C_{R}^{Q}}\left((1-2 \lambda) \xi^{\prime}(r)^{2}-2 \lambda \xi(r) \xi^{\prime \prime}(r)\right) d v_{h} .
\end{aligned}
$$

Using the definition of $\xi$ on $[R, Q]$ we obtain

$0 \leq-2 \lambda \xi(R) \xi^{\prime}(R) \ell(R)-2 \pi \lambda \xi(R)^{2}+\int_{C_{R}^{Q}}\left((1-4 \lambda) \alpha^{2}+2 \lambda \alpha\right)(Q-r)^{2 \alpha-2} d v_{h}$.

Since $1-4 \lambda<0$, we can choose $\alpha$ large enough so that $(1-4 \lambda) \alpha^{2}+2 \lambda \alpha \leq 0$, and we get

$$
0 \leq-2 \lambda \xi(R) \xi^{\prime}(R) \ell(R)-2 \pi \lambda \xi(R)^{2},
$$


which implies

$$
0 \leq-\frac{\xi^{\prime}(R)}{\xi(R)} \ell(R)-\pi=\frac{\alpha \ell(R)}{Q-R}-\pi .
$$

Since $M$ is complete, letting $Q$ tend to $+\infty$ we get the desired contradiction.

\section{The volume growth}

Proposition 2.2. Under the hypothesis of Theorem A, there exists a constant $c$ such that for all $R \geq 0$ we have $V(R) \leq c R^{2}$.

Proof. Let $Q>0$ and let $\xi:[0, Q] \rightarrow \mathbb{R}$ be the function defined by $\xi(s)=(Q-s)^{\alpha}$, where $\alpha \geq 1$ will be chosen later. Since $q_{\lambda}$ is nonnegative, we have

$$
0 \leq \int_{B_{Q}} \xi^{\prime}(r)^{2} d v_{h}+\lambda \int_{B_{Q}} \mathcal{K} \xi(r)^{2} d v_{h}
$$

Note that for all $s \geq 0$ we have $\chi\left(B_{s}\right) \leq 1$, so Lemma 1.8 gives

$$
\begin{aligned}
0 & \leq \int_{B_{Q}} \xi^{\prime}(r)^{2} d v_{h}+2 \pi \lambda \xi(0)^{2}-\lambda \int_{B_{Q}}\left(\xi^{2}\right)^{\prime \prime}(r) d v_{h} \\
& \leq 2 \pi \lambda Q^{2 \alpha}+\int_{B_{Q}}\left((1-4 \lambda) \alpha^{2}+2 \lambda \alpha\right)(Q-r)^{2 \alpha-2} d v_{h} .
\end{aligned}
$$

Note $A_{\alpha}=-\left((1-4 \lambda) \alpha^{2}+2 \lambda \alpha\right)$; since $1-4 \lambda<0$ we can choose $\alpha$ large enough so that $A_{\alpha}>0$. Thus we have the following inequalities:

$$
\begin{aligned}
A_{\alpha} \int_{B_{Q}}(Q-r)^{2 \alpha-2} d v_{h} & \leq 2 \pi \lambda Q^{2 \alpha}, \\
A_{\alpha} \int_{B_{\frac{Q}{2}}}(Q-r)^{2 \alpha-2} d v_{h} & \leq 2 \pi \lambda Q^{2 \alpha}, \\
A_{\alpha} \frac{Q^{2 \alpha-2}}{2^{2 \alpha-2}} V\left(\frac{Q}{2}\right) & \leq 2 \pi \lambda Q^{2 \alpha} .
\end{aligned}
$$

It follows that for all $R$ we have

$$
V(R) \leq \frac{2^{2 \alpha+1} \pi \lambda}{A_{\alpha}} R^{2} .
$$

The conformal type of the ends. It is a classical fact in potential theory that surfaces with quadratic volume growth are parabolic (i.e. each end is conformally equivalent to the punctured disc). For sake of completeness we give a proof of this fact which uses Proposition 1.9. 
Proposition 2.3. Under the hypothesis of Theorem A, each end of $M$ is conformally equivalent to the punctured disc $D^{*}=\{z \in \mathbb{C}|0<| z \mid<1\}$.

Proof. Suppose that $M$ possesses an end $E$ which is not conformally equivalent to $D^{*}$. Then there exists $a<1$ and a conformal diffeomorphism $F: E \rightarrow D_{a}=\{z \in \mathbb{C} \mid$ $a<|z|<1\}$.

Let $h_{\mathbb{H}^{2}}$ be the Poincaré metric on $D$, and let $h_{0}=F^{*}\left(h_{\mathbb{H}^{2}}\right)$. The diffeomorphism $F$ being conformal, and the metric $h_{\mathbb{H}^{2}}$ being conformal to the Euclidean one on $D$, we have $h=\mu^{2} h_{0}$ on $E$.

Let $\phi: M \rightarrow \mathbb{R}$ be the function defined by

$$
\phi(x)= \begin{cases}0 & \text { if } x \in M \backslash E \\ \frac{1}{4 \mu(x)^{2}} & \text { if } x \in E .\end{cases}
$$

Let $p$ be the quadratic form associated to the operator $\Delta-\phi$; for all functions $u \in C_{0}^{\infty}(M)$ we have

$$
p(u)=\int_{M}\left(|d u|_{h}^{2}-\phi u^{2}\right) d v_{h}=\int_{M \backslash E}|d u|_{h}^{2} d v_{h}+\int_{E}\left(|d u|_{h}^{2}-\frac{1}{4 \mu^{2}} u^{2}\right) d v_{h} .
$$

Using the conformal invariance of the Dirichlet integral in dimension 2 and the conformal diffeomorphism $F$, we get

$$
p(u)=\int_{M \backslash E}|d u|_{h}^{2} d v_{h}+\int_{D_{a}}\left(\left|d\left(u \circ F^{-1}\right)\right|_{h_{\mathbb{H}^{2}}}^{2}-\frac{1}{4}\left(u \circ F^{-1}\right)^{2}\right) d v_{h_{\mathbb{H}^{2}}} .
$$

It is a well-known fact that the second term of this sum is nonnegative.

We proved that there exists a function $\phi \in L_{\text {loc }}^{1}(M)$ which is nonnegative on $M$ and positive on a subset of positive measure, such that the operator $\Delta-\phi$ is nonnegative. Since $M$ has quadratic volume growth, this contradicts Proposition 1.9.

\section{A theorem "à la Huber"}

Let $(M, h)$ be a Riemannian surface satisfying the hypothesis of Theorem B: there exists a compact domain $\Omega \in M$ and a real $\lambda>\frac{1}{4}$ such that $q_{\lambda}(u) \geq 0$ for every function $u \in C_{0}^{\infty}(M \backslash \Omega)$. Let $R_{0}$ such that $\Omega \subset B_{R_{0}}$; the quadratic form $q_{\lambda}$ is nonnegative on $C_{0}^{\infty}\left(M \backslash B_{R_{0}}\right)$.

The proof of Theorem B is similar to the one of Theorem A. The difference is that we have to construct test functions whose supports are in $M \backslash B_{R_{0}}$. Choose $R_{1}$ such 
that $R_{0}<R_{1}-1$, and define $\xi_{0}:\left[R_{1}-1, R_{1}\right] \rightarrow \mathbb{R}$ by $\xi_{0}(s)=s-R_{1}+1$. In the sequel we shall note

$$
c_{h, \lambda}=\int_{C_{R_{1}-1}^{R_{1}}}\left(\xi_{0}^{\prime}(r)^{2}+\lambda \mathcal{K} \xi_{0}(r)^{2}\right) d v_{h}-\lambda G\left(R_{1}\right),
$$

which only depends on the metric $h$ and on $\lambda$.

Proposition 3.1. Under the hypotheses of Theorem B, M is of finite topology.

Proof. Suppose that the topology of $M$ is not finite. Let $A$ be such that $0>2 \pi A \lambda+$ $c_{h, \lambda}$; by Lemma 1.4, there exists $R$ such that for all $s \geq R$ we have $\chi\left(B_{s}\right) \leq A$.

Let $Q>R$ and let $\xi:\left[R_{1}-1, Q\right] \rightarrow \mathbb{R}$ be the function defined by

$$
\xi(s)= \begin{cases}\xi_{0}(s) & \text { for all } s \in\left[R_{1}-1, R_{1}[\right. \\ 1 & \text { on }\left[R_{1}, R[\right. \\ \frac{(Q-s)^{\alpha}}{(Q-R)^{\alpha}} & \text { for all } s \in[R, Q]\end{cases}
$$

where $\alpha \geq 1$ will be chosen later.

Since the quadratic form $q_{\lambda}$ is nonnegative on $C_{0}^{\infty}\left(M \backslash B_{R_{0}}\right)$, we have

$$
\begin{aligned}
0 \leq & \int_{C_{R_{1}-1}^{R_{1}}}\left(\xi_{0}^{\prime}(r)^{2}+\lambda \mathcal{K} \xi_{0}(r)^{2}\right) d v_{h}+\lambda \int_{C_{R_{1}}^{R}} \mathcal{K} d v_{h}+\int_{C_{R}^{Q}} \xi^{\prime}(r)^{2} d v_{h} \\
& +\lambda \int_{C_{R}^{Q}} \mathcal{K} \xi(r)^{2} d v_{h} \\
\leq & c_{h, \lambda}+\lambda G(R)+\int_{C_{R}^{Q}} \xi^{\prime}(r)^{2} d v_{h}+\lambda \int_{C_{R}^{Q}} \mathcal{K} \xi(r)^{2} d v_{h} .
\end{aligned}
$$

Since $\chi\left(B_{s}\right) \leq A$ for all $s \in[R, Q]$, Lemma 1.8 gives

$$
\begin{aligned}
0 \leq c_{h, \lambda} & +\lambda G(R)+\int_{C_{R}^{Q}} \xi^{\prime}(r)^{2} d v_{h}-\lambda G(R)-2 \lambda \xi^{\prime}(R) \ell(R)+2 \pi A \lambda \\
& -\lambda \int_{C_{R}^{Q}}\left(\xi^{2}\right)^{\prime \prime}(r) d v_{h} .
\end{aligned}
$$

Using the definition of $\xi$ on $[R, Q]$ we get

$0 \leq c_{h, \lambda}+2 \pi A \lambda+\frac{2 \alpha \lambda \ell(R)}{Q-R}+\frac{1}{(Q-R)^{2 \alpha}} \int_{C_{R}^{Q}}\left((1-4 \lambda) \alpha^{2}+2 \lambda \alpha\right)(Q-r)^{2 \alpha-2} d v_{h}$.

Since $1-4 \lambda<0$, we can choose $\alpha$ large enough so that $(1-4 \lambda) \alpha^{2}+2 \lambda \alpha \leq 0$, and letting $Q$ tend to $+\infty$ we get $0 \leq c_{h, \lambda}+2 \pi A \lambda$ which is in contradiction with the choice of $A$. 
Proposition 3.2. Under the hypotheses of Theorem B, there exists a constant $c$ such that for all $R \geq 0$ we have $V(R) \leq c R^{2}$.

Proof. Let $Q>R_{1}$ and let $\xi:\left[R_{1}-1, Q\right] \rightarrow \mathbb{R}$ be the function defined by

$$
\xi(s)= \begin{cases}\xi_{0}(s) & \text { for all } s \in\left[R_{1}-1, R_{1}[;\right. \\ \frac{(Q-s)^{\alpha}}{\left(Q-R_{1}\right)^{\alpha}} & \text { for all } s \in\left[R_{1}, Q\right] .\end{cases}
$$

where $\alpha \geq 1$ will be chosen later.

The nonnegativity of $q_{\lambda}$ gives

$$
0 \leq \int_{C_{R_{1}-1}^{R_{1}}}\left(\xi_{0}^{\prime}(r)^{2}+\lambda \mathcal{K} \xi_{0}(r)^{2}\right) d v_{h}+\int_{C_{R_{1}}^{Q}} \xi^{\prime}(r)^{2} d v_{h}+\lambda \int_{C_{R_{1}}^{Q}} \mathcal{K} \xi(r)^{2} d v_{h} .
$$

Since for all $s \geq 0$ we have $\chi\left(B_{s}\right) \leq 1$, Lemma 1.8 yields

$$
\begin{aligned}
0 & \leq c_{h, \lambda}-2 \lambda \xi^{\prime}\left(R_{1}\right) \ell\left(R_{1}\right)+2 \pi \lambda+\int_{C_{R_{1}}^{Q}} \xi^{\prime}(r)^{2} d v_{h}-\lambda \int_{C_{R_{1}}^{Q}}\left(\xi^{2}\right)^{\prime \prime}(r) d v_{h} \\
& \leq c_{h, \lambda}+2 \pi \lambda+\frac{2 \alpha \lambda \ell\left(R_{1}\right)}{Q-R_{1}}-\frac{A_{\alpha}}{\left(Q-R_{1}\right)^{2 \alpha}} \int_{C_{R_{1}}^{Q}}(Q-r)^{2 \alpha-2} d v_{h}
\end{aligned}
$$

where $A_{\alpha}=-\left((1-4 \lambda) \alpha^{2}+2 \lambda \alpha\right)$; since $1-4 \lambda<0$ we can choose $\alpha$ large enough so that $A_{\alpha}>0$. Thus we have the following inequalities:

$$
\begin{gathered}
\frac{A_{\alpha}}{\left(Q-R_{1}\right)^{2 \alpha}} \int_{C_{R_{1}}^{Q}}(Q-r)^{2 \alpha-2} d v_{h} \leq c_{h, \lambda}+2 \pi \lambda+\frac{2 \alpha \lambda \ell\left(R_{1}\right)}{Q-R_{1}}, \\
\frac{A_{\alpha}\left(Q+R_{1}\right)^{2 \alpha-2}}{2^{2 \alpha-2}\left(Q-R_{1}\right)^{2 \alpha}} \operatorname{Vol}\left(C_{R_{1}}^{\frac{R_{1}+Q}{2}}\right) \leq c_{h, \lambda}+2 \pi \lambda+\frac{2 \alpha \lambda \ell\left(R_{1}\right)}{Q-R_{1}} .
\end{gathered}
$$

Let $R>R_{1}$ and let $Q>R_{1}$ such that $R=\frac{R_{1}+Q}{2}$. It follows from the above inequalities that

$V(R)=V\left(R_{1}\right)+\operatorname{Vol}\left(C_{R_{1}}^{R}\right) \leq V\left(R_{1}\right)+\left(c_{h, \lambda}+2 \pi \lambda+\frac{\alpha \lambda \ell\left(R_{1}\right)}{R-R_{1}}\right) \frac{2^{2 \alpha}\left(R-R_{1}\right)^{2 \alpha}}{A_{\alpha} R^{2 \alpha-2}}$.

From this inequality we deduce that $\frac{V(R)}{R^{2}}$ is bounded above on $\left[R_{1}+1,+\infty\right)$; since $\lim _{R \rightarrow 0} \frac{V(R)}{R^{2}}=\pi, \frac{V(R)}{R^{2}}$ is bounded above on $\mathbb{R}$, which implies the result.

Proposition 3.3. Under the hypotheses of Theorem B, each end of $M$ is conformally equivalent to the punctured disc.

Proof. The proof is the same as the one of Proposition 2.3.

Remark 3.4. The hypotheses of Theorem B are satisfied if there exists $\lambda>\frac{1}{4}$ such that the operator $\Delta+\lambda \mathcal{K}$ as a finite number of negative eigenvalues. 


\section{Complete conformal metrics on the unit disc}

In the following, a "complete conformal metric on the unit disc" will denote a metric $h$ on $D=\{z \in \mathbb{C}|| z \mid<1\}$ which is complete and conformally equivalent to the Euclidean metric.

In this section, we are interested in the question asked in [FC-Sc]: If $h$ is a complete conformal metric on the unit disc, what are the possible values of $b_{h}$ which can occur? Since the unit disc is not conformally equivalent to $\mathbb{C}$ nor $\mathbb{C}^{*}$, Theorem A gives a first answer to this question.

Corollary 4.1. If $h$ is a complete conformal metric on the unit disc, then $b_{h} \leq \frac{1}{4}$.

Remark 4.2. The statement of Theorem A is the best possible since $b_{h}=\frac{1}{4}$ for the Poincaré metric on $D$. Moreover, $\mathbb{C}$ and $\mathbb{C}^{*}$ admit complete flat metrics wich are conformally equivalent to the Euclidean one. For such metrics we have $b_{g}=+\infty$.

It is not hard to see that $b_{h}$ can take any value in $\left[0, \frac{1}{4}\right]$ for a complete conformal metric $h$ on the unit disc. Namely, we have the following proposition.

Proposition 4.3. Let $h=\mu^{2}|d z|^{2}$ be a complete conformal metric on the unit disc. If the metric $h_{\alpha}=\mu^{2 \alpha}|d z|^{2}$ is complete, then we have $b_{h_{\alpha}}=\frac{1}{\alpha} b_{h}$

Proof. Let $q_{\lambda, h}$ (resp. $q_{\lambda, h_{\alpha}}$ ) denote the quadratic form associated to the operator $\Delta+\lambda \mathcal{K}$ for the metric $h$ (resp. $h_{\alpha}$ ). Using the conformal invariance of the Dirichlet integral, and the expressions of the curvature and the volume form of $h$ in terms of the conformal factor $\mu$, we get

$$
q_{\lambda, h}(u)=\int_{D}\left(|d u|_{e}^{2}+\lambda(\Delta \log \mu) u^{2}\right) d v_{e},
$$

where $|d u|_{e}$ and $d v_{e}$ denote the norm of $d u$ and the volume form for the Euclidean metric.

A similar calculation gives

$$
q_{\lambda, h_{\alpha}}(u)=\int_{D}\left(|d u|_{e}^{2}+\alpha \lambda(\Delta \log \mu) u^{2}\right) d v_{e}
$$

so that $q_{\lambda, h_{\alpha}}=q_{\alpha \lambda, h}$ and the proposition follows.

If $h$ is the Poincaré metric (i.e. $\mu(z)=\frac{2}{1-|z|^{2}}$ ), and if $\alpha \geq 1$, then it is easy to see that the metric $h_{\alpha}=\mu^{2 \alpha}|d z|^{2}$ is complete, so we have $b_{h_{\alpha}}=\frac{1}{4 \alpha}$. 
Consider now the metric $h=\mu^{2}|d z|^{2}$, with $\mu(z)=\exp \left(\frac{1}{1-|z|^{2}}\right)$. Let $R<1$ and let $\xi_{R}:[0,1] \rightarrow \mathbb{R}$ be the function defined by

$$
\xi_{R}(s)= \begin{cases}1 & \text { if } s \leq R \\ \frac{R+1-2 s}{1-R} & \text { if } R \leq s \leq \frac{R+1}{2} \\ 0 & \text { if } s \geq \frac{R+1}{2}\end{cases}
$$

Then a simple calculation proves that, for all $\lambda>0, q_{\lambda, h}\left(\xi_{R}(|z|)\right)$ becomes negative if $R$ is close enough to 1 . This implies that $b_{h}=0$.

Finally, for each $\beta \in\left[0, \frac{1}{4}\right]$ we found a complete conformal metric $h$ on $D$ such that $b_{h}=\beta$.

Another natural question is to know whether the value $b_{h}=\frac{1}{4}$ is characteristic of the Poincaré metric among the complete conformal metrics on the unit disc. The (negative) answer is given by the following proposition.

Proposition 4.4. There exists a universal constant $\varepsilon$ such that for all complete conformal metrics $h$ on the unit disc satisfying $\mathcal{K} \leq-1$ and $\int_{D}|\mathcal{K}+1|^{\frac{3}{2}} d v_{h} \leq \varepsilon$ we have $b_{h}=\frac{1}{4}$.

Proof. The surface being simply connected, the upper bound on the curvature implies an upper bound on the heat kernel $p_{h}$ of $(D, h)$. This is obtained by comparing $p_{h}$ with the hyperbolic heat kernel, using standard comparison theorems; using known estimates on the hyperbolic heat kernel, we get that there exists a universal constant $A_{0}$ such that $p_{h}(t, x, x) \leq A_{0} t^{-\frac{3}{2}} \mathrm{e}^{-\frac{t}{4}}$ (cf. for example [Ca1], Proposition 2.4).

Using Lieb's theorem (cf. [Ca1], Theorem 1.3), there exists a universal constant $A$ such that for every operator of the type $R=\Delta-\frac{1}{4}+\phi$, the number of negative eigenvalues of $R$ satisfies $\mathcal{N}_{0}(R) \leq A \int_{D}|\phi|^{\frac{3}{2}} d v_{h}$. Let $\varepsilon=\frac{1}{A}$, and suppose $\int_{D}|\mathcal{K}+1|^{\frac{3}{2}} d v_{h} \leq \varepsilon$. Since the operator $L_{\frac{1}{4}}$ is $L_{\frac{1}{4}}=\Delta+\frac{1}{4} \mathcal{K}=\Delta-\frac{1}{4}+\frac{1}{4}(\mathcal{K}+1)$, we have

$$
\mathcal{N}_{0}\left(L_{\frac{1}{4}}\right) \leq \frac{A}{8} \int_{D}|\mathcal{K}+1|^{\frac{3}{2}} d v_{h} \leq \frac{1}{8} .
$$

This inequality implies that $\mathcal{N}_{0}\left(L_{\frac{1}{4}}\right)=0$, so $q_{\frac{1}{4}}$ is non negative and $b_{h} \geq \frac{1}{4}$. Using Corollary 4.1 we get $b_{h}=\frac{1}{4}$.

Remark 4.5. The hypotheses of Proposition 4.4 are satisfied by some of the minimal surfaces of $\mathbb{H}^{3}$ introduced in [Ne-Sp]. For some simply connected domain $\Omega \subset \mathbb{R}^{2}$, the authors prove that there exists a function $f_{\Omega}$ which is zero on $\partial \Omega$ and positive on $\Omega$ whose graph $M_{\Omega}$ is a minimal surface in the upper half space endowed with its hyperbolic metric (when $\Omega$ is a Euclidean ball, $M_{\Omega}$ is a hyperbolic plane). 
Now, the Gauss equation implies that $\mathcal{K} \leq-1$ on $M_{\Omega}$, and taking $\Omega$ close enough to a Euclidean ball, it is not hard to see that $\int_{M_{\Omega}}|\mathcal{K}+1|^{\frac{3}{2}} d v_{h}$ can be made small enough to fit the hypothesis of Proposition 4.4.

\section{References}

[Bé-Be] P. Bérard, G. Besson, Number of bound states and estimates on some geometric invariants. J. Funct. Anal. 94 (1990), 375-396. Zbl 0723.53026 MR 1081650

[Ca1] P. Castillon, Problèmes de petites valeurs propres sur les surfaces de courbure moyenne constante. Proc. Amer. Math. Soc. 130 (2002), 1153-1163. Zbl 01721011 MR 1873791

[Ca2] P. Castillon, Spectral properties and conformal type of surfaces. An. Acad. Brasil. Ciênc. 74 (2002), 585-588. Zbl 1031.53060 MR 1942926

[Co-Mi] T. Colding, W. Minicozzi, Estimates for parametric elliptic integrands. Internat. Math. Res. Notices 2002 (2002), 291-297. Zbl 1002.53035 MR 1877004

[Fi] F. Fiala, Le problème des isopérimètres sur les surfaces ouvertes à courbure positive. Comment. Math. Helv. 13 (1940), 293-346. Zbl 0025.23003 MR 0006422

[FC-Sc] D. Fischer-Colbrie, R. Schoen, The structure of complete stable minimal surfaces in 3-manifolds of non-negative scalar curvature. Comm. Pure Appl. Math. 33 (1980), 199-211. Zbl 0439.53060 MR 0562550

[Ha] P. Hartman, Geodesic parallel coordinates in the large. Amer. J. Math. 86 (1964), 705-727. Zbl 0128.16105 MR 0173222

[Hu A. Huber, On subharmonic functions and differential geometry in the large. Comment. Math. Helv. 32 (1957), 13-72. Zbl 0080.15001 MR 0094452

[Ne-Sp] B. Nelli, J. Spruck, On the existence and uniqueness of constant mean curvature hypersurfaces in hyperbolic space. In Geometric analysis and the calculus of variation (J. Jost, ed.), International Press, Cambridge, MA (1996) 253-266. Zbl 0936.35069 MR 1449411

[Ri] I. Richards, On the classification of non-compact surfaces. Trans. Amer. Math. Soc. 106 (1963), 259-269. Zbl 0156.22203 MR 0143186

[Sh-Ta1] K. Shiohama, M. Tanaka, An isoperimetric problem for infinitely connected complete open surfaces. In Geometry of manifolds (Matsumoto, 1988), Perspect. Math. 8, Academic Press, Boston, MA, 1989, 317-343. Zbl 0697.53040 MR 1040533

[Sh-Ta2] K. Shiohama, M. Tanaka, The length function of geodesic paralel circle. In Progress in differential geometry (K. Shiohama, ed.), Adv. Stud. Pure Math. 22, Math. Soc. Japan, Tokyo 1993, 299-308. Zbl 0799.53052 MR 1274955

Received June 7, 2004

Philippe Castillon, i3m (u.m.r., c.n.r.s. 5149), Départment des Sciences Mathématiques, CC 5, Université Montpellier II, 34095 Montpellier Cedex 5, France

E-mail: cast@math.univ-montp2.fr 